\title{
BMJ Open Diagnosis and management of surgical disease at Ethiopian health centres: cross-sectional survey of resources and barriers to care
}

\author{
Nichole Starr (D) , ${ }^{1}$ Sarah Carpenter, ${ }^{2}$ Melissa Carvalho, ${ }^{1}$ Aileen Souza, ${ }^{3}$ \\ Robin Chin, ${ }^{2}$ George Kasotakis, ${ }^{4}$ Mengistu Worku ${ }^{5}$
}

To cite: Starr N, Carpenter S, Carvalho M, et al. Diagnosis and management of surgical disease at Ethiopian health centres: cross-sectional survey of resources and barriers to care. BMJ Open 2019;9:e031525. doi:10.1136/ bmjopen-2019-031525

- Prepublication history for this paper is available online. To view these files, please visit the journal online (http://dx.doi. org/10.1136/bmjopen-2019031525).

Received 10 May 2019 Revised 03 September 2019 Accepted 30 September 2019

Check for updates

(C) Author(s) (or their employer(s)) 2019. Re-use permitted under CC BY-NC. No commercial re-use. See rights and permissions. Published by BMJ.

${ }^{1}$ Department of Surgery, University of California, San Francisco, San Francisco, California, USA

${ }^{2}$ School of Medicine, Boston University, Boston, Massachusetts, USA

${ }^{3}$ Department of Pediatrics, Brown University, Providence, Rhode Island, USA

${ }^{4}$ Surgery, Duke University, Durham, North Carolina, USA

${ }^{5}$ Department of Surgery, Dessie Referral Hospital, Dessie, Ethiopia

Correspondence to

Dr Nichole Starr;

nichole.starr@ucsf.edu

\section{ABSTRACT}

Objectives The aim of this study was to characterise the resources and challenges for surgical care and referrals at health centres (HCs) in South Wollo Zone, Ethiopia.

Setting Eight primary HCs in South Wollo Zone, Ethiopia. Participants Eight health officers and nurses staffing eight HCs completed a survey.

Design The study was a survey-based, cross-sectional assessment of HCs in South Wollo Zone, Ethiopia and data were collected over a 30-day period from November 2014 to January 2015.

Primary and secondary outcome measures Survey assessed human and material resources, diagnostic capabilities and challenges and patient-reported barriers to care.

Results Eight HCs had an average of 18 providers each, the majority of which were nurses $(62.2 \%)$ and health officers (20.7\%). HCs had intermittent availability of clean water, nasogastric tubes, rectal tubes and suturing materials, none of them had any form of imaging. A total of 168 surgical patients were seen at the $8 \mathrm{HCs} ; 58 \%$ were referred for surgery. Most common diagnoses were trauma/burns (42\%) and need for caesarean section (9\%). Of those who did not receive surgery, 32 patients reported specific barriers to obtaining care (91.4\%). The most common specific barriers were patients not being decision makers to have surgery, lack of family/social support and inability to afford hospital fees.

Conclusions HCs in South Wollo Zone, Ethiopia are wellstaffed with nurses and health officers, however they face a number of diagnostic and treatment challenges due to lack of material resources. Many patients requiring surgery receive initial diagnosis and care at HCs; sociocultural and financial factors commonly prohibit these patients from receiving surgery. Further study is needed to determine how such delays may impact patient outcomes. Improving material resources at $\mathrm{HCs}$ and exploring community and family perceptions of surgery may enable more streamlined access to surgical care and prevent delays.

\section{INTRODUCTION}

Global health priorities have evolved over the past several decades with a greater focus placed on surgery as part of a package of essential healthcare. Estimates indicate that
Strengths and limitations of this study

This study is the first of its kind to address the limitations at the health centre level in Ethiopia which may present barriers to surgical access.

- Our survey was conducted with healthcare providers in the South Wollo Zone of Ethiopia, who reported their perspectives on training and diagnostic abilities, as well as facility level material resources, patient mix and volume.

- In the context of the ongoing Saving Lives Through Safer Surgery National Surgical Plan of Ethiopia, this study highlights important patient and provider-related challenges that can be considered in the strengthening of Ethiopian surgical referral system.

- This survey incorporates both training and resource limitations, as well as patient-reported barriers to surgical access, that capture the complex nature of the surgical referral ecosystem, particularly in rural areas.

- As the sample size was low, results may not be generalisable to other parts of the region or country in Ethiopia, but represent important perspectives that can inform future study.

up to $30 \%-35 \%$ of the global burden of disease could be treated with surgical interventions. ${ }^{1-3}$ However, there is great inequity in the distribution of surgical care worldwide, with $73.6 \%$ of surgical procedures performed on $30 \%$ of the global population, and the poorest third receiving only $3.5 \%$ of surgeries. ${ }^{4}$ Many studies have aimed to quantify the burden of surgical disease and describe existing resources and infrastructure related to surgical care worldwide, in an effort to best direct interventions and improve access. It is clear that there is a shortage of trained personnel and equipment at the district hospital level to provide adequate surgical services in low and middle-income countries. ${ }^{5}$ 
Ethiopia is particularly affected by these shortages. A survey of surgical services in 2012 found only 106 general surgeons in 116 federal hospitals serving a population of 82.8 million. ${ }^{6}$ Although the surgical workforce has increased in recent years with the expansion of residency training programmes and task-shifting to Integrated Emergency Surgical Officers (IESOs)—clinicians with additional emergency surgical training-there is still a shortage of surgical and anaesthesia care providers. Additionally hospitals are not well resourced with limited capacity to provide blood transfusions, supplemental oxygen and adequate patient monitoring at all facilities. ${ }^{7}$

Access to the 'Bellwether procedures', debridement of open fracture, emergency laparotomy and caesarean section within 2 hours for $80 \%$ of the population is a target established by the Lancet Commission on Global Surgery, with target procedure volumes of 5000/100 000 population. ${ }^{5}$ Out of 56 WHO member states investigated in a study focused on documented rates of surgery, Ethiopia had the lowest surgical volume rate at 148 per $100000 .^{8}$ Part of this phenomenon may be explained by the limited resources available for surgical interventions in Ethiopia. Hospitals often lack reliable access to running water, medications, electricity, oxygen and blood banking. The average hospital had a maximum of two operating rooms for the vast population served. ${ }^{6}$

The number of surgeons and other health workers within local hospitals or clinics is limited as well. A retrospective survey study conducted to outline surgical activity in rural Ethiopia found 76 health workers able to provide surgical services to 12.9 million people made up the sample population. ${ }^{9}$

Beyond the lack of hospital equipment and personnel, however, other factors impede the ability of patients to access surgical services. Community assessments have revealed that cultural, financial and structural barriers impact the ability of patients to seek and access care. ${ }^{10}$ Many patient factors are barriers to obtaining care, such as fear of surgery, lack of time or monetary resources, lack of transportation, cultural beliefs surrounding surgery, health illiteracy and lack of social support systems. Structural barriers such as poor roads, distance to hospitals, lack of communication and difficulty navigating the healthcare and referral system also impede the ability of patients to access surgical care. Medical services and loss of wages may be prohibitively expensive for most patients. These populations also tend to be spread across rural areas in Ethiopia, and have difficulty reaching hospitals in larger cities. ${ }^{11}$

Mid-level and rural healthcare providers have the unique position as both the gatekeeper and facilitator between patients and facilities capable of providing surgical care. Few studies, however, have examined the barriers perceived by providers in the process of obtaining surgical care. ${ }^{12}$

For the majority of Ethiopians, the nearest healthcare facility would be a town or city health centre (HC). Patients needing urgent medical attention may seek initial care at these HCs, after which they may be referred for appropriate higher level of care. According to the 2012 Ethiopian Standard Health Center Requirements, ${ }^{13}$ recommended practitioners at each HC include a minimum of two health officers, three midwives, five nurses and laboratory and pharmacy technicians. The guidelines also state each HC should have the capacity to perform minor operations such as circumcision, lipoma excision and abscess drainage and external immobilisation of fractures.

\section{The SaLTS programme}

Contemporaneously with this study, the Ethiopian Federal Ministry of Health and international partner organisations began the 'Saving Lives through Safer Surgery' (SaLTS) Initiative, and multipronged national surgical plan to improve access to and quality of surgical care throughout Ethiopia. ${ }^{14}$ This initiative not only better characterised resources and surgical volume at hospitals, but also developed a nationwide plan to improve surgical leadership, infrastructure, data quality, human and materials resources. The SaLTS initiative does not specifically outline a plan for HCs in the surgical referral network, however SaLTS does prioritise access, quality and surgical volume as targets for the programme. ${ }^{14}$

As part of the SaLTS initiative to increase access to timely, safe and affordable surgical care when needed in Ethiopia, HCs are an important piece in the referral network that can serve the role of decreasing burden on hospitals by managing minor conditions and efficiently referring patients to hospital when surgery is needed. However, in order to play a role in increasing surgical access and safety in Ethiopia, HCs must be consistently equipped with necessary material and human resources, and must have the training to confidently diagnose, triage and at times manage surgical conditions.

As investment in surgical services is expanding in Ethiopia, it is important to understand the material and human capabilities of the HCs which are often responsible for the diagnosis and referral of surgical conditions. Additionally, with the possible expansion of surgical services to more rural and primary healthcare facilities in Ethiopia, it is important to understand the current available human and material resources, as well as patient mix presenting to those facilities.

The 'three delays' model of accessing care has often been used to describe delays in care contributing to maternal mortality at several time points, the first being decision to seek healthcare, the second reaching the appropriate medical facility and the third receiving timely, appropriate intervention after reaching the right facility. ${ }^{15}$ This model is also applicable to emergency surgical and trauma care, when timely access to higher-level care is often critical for patient survival. ${ }^{16}$ Barriers faced by patients and their families can contribute to care-seeking behaviours in the first delay; lack of resources or training at intermediate facilities such as HCs can lead to 'third delays' in care. 
The aim of this study was to describe material and human resources available at HCs in the South Wollo Zone, Ethiopia and assess barriers experienced by mid-level providers and patients at HCs in obtaining surgical care.

\section{METHODS}

\section{Study setting}

Ethiopia is a low-income country in Eastern Sub-Saharan Africa with a rapidly growing population. With a population of 102 million in 2016, life expectancy was estimated as 65.5 years. ${ }^{17}$ Maternal mortality is moderate at $353 / 100000$ live births, and under age 5 mortality rate is rapidly falling, estimated as 61/100 000 in 2015. Surgical diseases, however, are now a major source of mortality, with non-communicable diseases, particularly cardiovascular disease, malignant neoplasms and digestive diseases, now making up a majority of causes of death. It was reported that only $55 \%$ of healthcare facilities could provide basic obstetric care and $52 \%$ could provide basic surgical care in 2015 .

The ratio of healthcare providers and facilities per population is among the lowest in the world, with 149 total hospitals in country, $1343 \mathrm{HCs}$ and 3305 health posts in 2008. This study was conducted in the Amhara region, which, at last regional census, was estimated to have 17.2 million inhabitants in 2007 with 7406 total healthcare providers, 2152 physicians $(2.7 / 100000 \mathrm{popu}-$ lation). In the most recent available provider densities in 2007, an estimated 140 surgeons were practicing in the country, with estimates for 820 by $2015 .^{17}$

Dessie Referral Hospital is the only referral hospital in South Wollo Zone within the Amhara Region (figure 1) and serves an estimated catchment area of 7 million, with general surgeons, orthopaedic surgeons and obstetricians on staff. While intensive/critical care is limited, and subspecialties such as oncological care are not available, all Bellwether procedures can be performed at this hospital.

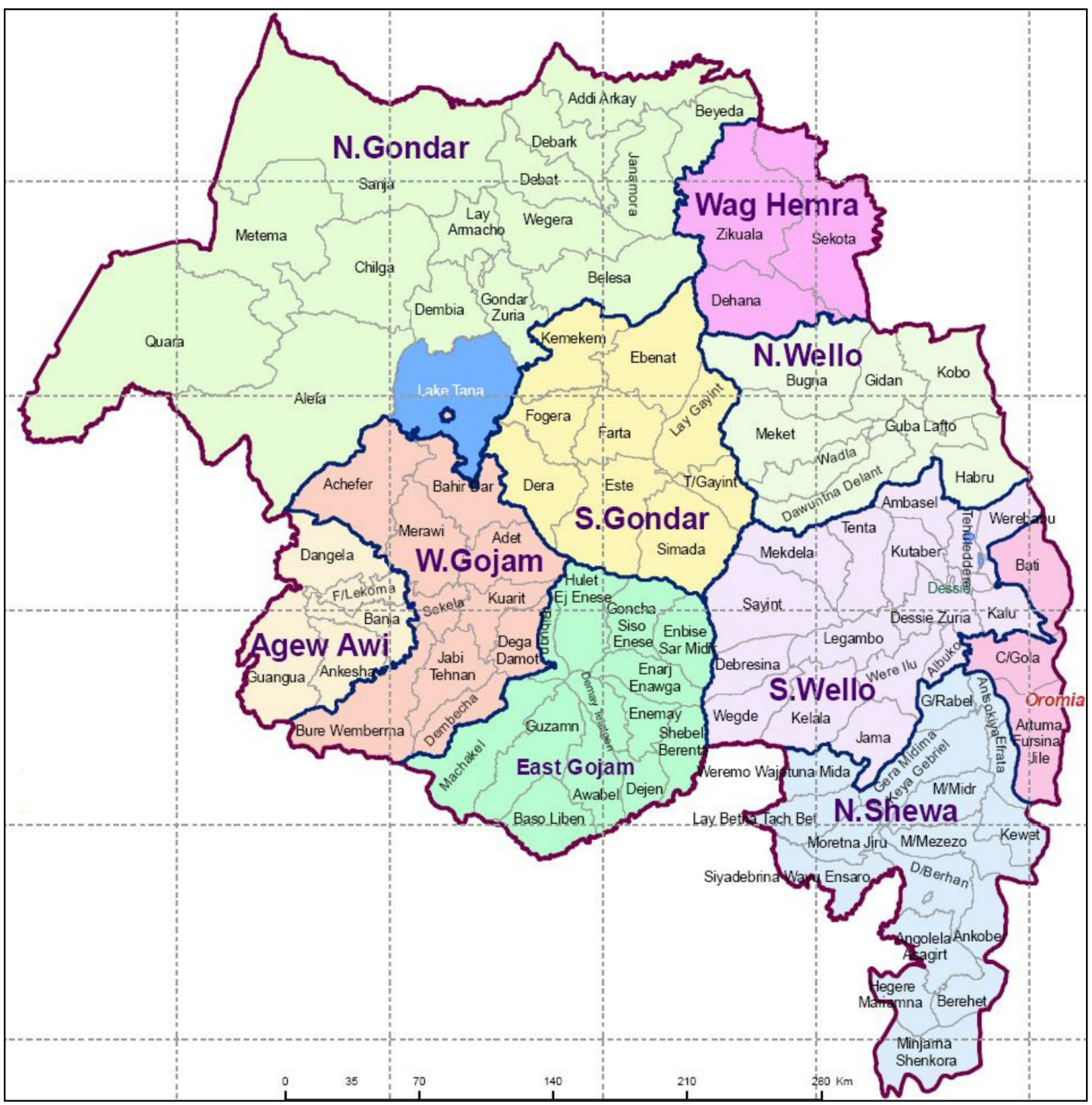

Figure 1 Amhara region and South Wollo zone map with study site locations. 
This study was a cross-sectional survey of eight woreda (district) level HCs in the South Wollo Zone of Ethiopia. Survey was designed by the study personnel using review of prior similar studies on barriers to accessing surgery and HC assessment tools. ${ }^{10}{ }^{18-21}$ Surveys were distributed to all HCs in the South Wollo Zone for completion. The survey was reviewed by local colleagues at the main study site at Dessie Referral Hospital in South Wollo Zone and feedback incorporated into the final tool. The survey tool was piloted with one $\mathrm{HC}$ in Dessie town with a surgeon and HC nurse and all questions were determined to be understandable and possible to answer by the local study personnel. Although Dessie Hospital is considered a 'referral hospital' by the Ethiopian healthcare network, according to international standards it meets criteria as a primary hospital, with inpatient and general surgical services available, but lacking subspecialty or intensive care unit services. ${ }^{22}$

\section{Patient and public involvement statement}

Patients and the public were not involved in the study design or survey tool design; however, local healthcare providers gave input on survey questions during study design. Findings from this study will be made available at the referral hospital for public viewing and dissemination.

\section{Participants}

Surveys were distributed at a regional health bureau meeting. Study participants who completed the survey were nurses or health officers (diploma nurses with additional training) employed at the respective HCs. Orientation and training on survey completion were provided by an Ethiopian nurse who was engaged in the project. This trainer and the principal investigator (PI) were available to answer questions for participants throughout the study period by phone regarding survey completion. The survey was distributed to HC nurses or health officers at all 21 woredas in South Wollo. Participation was voluntary and participants received a small monetary compensation when the survey was returned at the end of the study period.

\section{Variables}

Study participants completed survey questions regarding HC staffing, diagnostic and treatment resources available at their HC, as well as provider comfort level making common surgical diagnoses in their setting. They also recorded data on all patients presenting to their respective HCs with surgical diagnoses as stipulated by the study definitions over a 30-day period. Information about these patients including age, gender, diagnosis, whether or not a referral was made at the clinic visit and barriers expressed or perceived prevented patients from receiving surgical care were recorded.

\section{Data collection}

Participants collected patient data over a 30-day period between November 2014 and January 2015; HCs varied in the specific dates of their data collection. Surveys were returned to the study PI in person or via post and compensation was provided on return of completed survey. All participating HCs were in a geographical network making surgical referrals to a single referral hospital in Dessie, Ethiopia. In an effort to avoid selection bias, surveys were distributed to all HCs in the Zone and orientation to the survey was conducted with staff from all HCs. Study population included all HCs in South Wollo Zone which were expected to make referrals to a single hospital in the Zonal capital.

\section{Quantitative variables}

Quantitative data such as HC catchment population, staffing, patient age and referral status were analysed with frequencies and SD. Descriptive statistics were used for all variables and no multivariate analysis or associations were calculated.

Data were returned via paper forms and entered into RedCap by study personnel. Data were extracted to Microsoft Excel and kept confidential on encrypted computer by study personnel. Descriptive statistics were used to analyse HC providers, resources, diagnostic challenges, patient diagnoses and barriers to care. Data analysis was conducted using Excel and Stata/SE V. 15.1.

\section{RESULTS}

Eight HCs in South Wollo Zone returned surveys, representing $38 \%$ of the total zonal HCs $(n=21)$. HCs were distributed geographically throughout the zone with a mean distance of $93.9 \mathrm{~km} \pm 59.3 \mathrm{~km}$ (58.3 miles \pm 36.8 miles) from the Dessie Referral Hospital (figure 1). Each HC served a population of approximately 36000 in its catchment area. Given road quality and typical transport speeds, it would take an estimate of 2-4hours to reach the Referral Hospital from the average participating HC.

\section{Providers}

An average of $18( \pm 4)$ clinical service providers (physicians, health officers or nurses) were employed at each $\mathrm{HC}$ and saw an average of 1212 (range 278-4118) patients at each facility in the 30-day study period (table 1). On average, $25(2.1 \%)$ of these patients had a surgical complaint. Only one HC was staffed by physicians and none had anaesthesia providers. The majority of providers were health officers $(20.7 \%)$, nurses $(62.2 \%)$ and midwives $(13.7 \%)$. Half of the HCs had lab and pharmacy technicians.

\section{Resources}

All HCs had access to electricity, pain medications, antibiotics and antipyretics (figure 2). All but one centre had minor suturing materials. Half of centres reported that they always had access to nasogastric (NG) or rectal tubes. Only two centres always had clean water, while the other six reported that they sometimes had clean water. Only one reported access to blood for transfusion, and no HCs 
Table 1 Baseline characteristics of health centres $(n=8)$

\begin{tabular}{ll}
\hline Baseline characteristics & $\mathbf{N}(\%)$ \\
\hline $\begin{array}{l}\text { Distance from referral hospital }(\mathrm{km}) \\
\text { (mean } \pm \text { SD) }\end{array}$ & $93.9 \pm 59.3$ \\
$\begin{array}{l}\text { Woreda (administrative zone) population } \\
\text { (mean } \pm \text { SD) }\end{array}$ & $36300 \pm 19859$ \\
\hline $\begin{array}{l}\text { Total patients seen in } 30 \text { days (mean } \pm \text { SD) } \\
\text { Surgical patients seen in } 30 \text { days }\end{array}$ & $1212 \pm 1345$ \\
(mean \pm SD) & $25 \pm 7.5$ \\
\hline $\begin{array}{l}\text { Number of healthcare providers } \\
\text { (mean } \pm S D)\end{array}$ & $18 \pm 4$ \\
\hline
\end{tabular}

Frequency of providers at all health centres

$\begin{array}{ll}\text { Physicians } & \\ \text { None } & 6 \\ 1-5 & 0 \\ 5-10 & 1 \\ >10 & 0\end{array}$

\section{Health officers}

$\begin{array}{ll}1-5 & 6 \\ 5-10 & 1 \\ >10 & 1 \\ \text { Nurses and nursing assistants } & \\ 1-5 & - \\ 5-10 & 5 \\ >10 & 3\end{array}$

\section{Anaesthetists}

None 8

Midwives

1-5 8

Lab technician

\begin{tabular}{ll} 
None & 4 \\
$1-5$ & 4 \\
Pharmacy technician & \\
None & 4 \\
$1-5$ & 4 \\
\hline
\end{tabular}

had access to any form of imaging (ultrasound, radiograph or CT scan) or paracentesis kits.

\section{Diagnostic capabilities}

Providers reported the difficulty of diagnosing common illnesses with their available resources (figure 3). According to $\mathrm{HC}$ provider ratings, the most difficult diagnoses were cholecystitis and other gallstone disease, intra-abdominal tumours, perforated ulcer and kidney stones. Most often lack of diagnostic aids was cited as the reason for difficulty. The conditions most easily diagnosed were traumatic injury, appendicitis, a need for caesarean section, hernia, skin/soft tissue tumours and peptic ulcer disease (table 2). Even for the diagnoses that were rated as 'not difficult at all', lack of diagnostic aids and lack of training were still cited as reasons for difficulty.

\section{Surgical patients}

A total of 168 patients were seen with surgical complaints over the 30-day study period at the 8 HCs (table 3). Patients' average age was 34 , ranging $1-80$ years and $63.6 \%$ were men. Ninety-seven patients $(58 \%)$ were referred to the hospital for surgery; of the 71 patients $(42 \%)$ not referred for surgery, the majority of them $(87 \%)$ could be treated in clinic. The other nine $(12.6 \%)$ refused referral. Most surgical patients seen in HCs had traumatic injuries or burns (42\%) (figure 4). Other common diagnoses seen in the HCs were need for caesarean section (9\%), appendicitis $(5 \%)$, benign prostatic hyperplasia $(4 \%)$, peptic ulcer disease $(6 \%)$ and bowel obstruction $(5 \%)$. Less common diagnoses were kidney stones, soft tissue infections, goitres, haemorrhoids, requests for circumcision and soft tissue tumours.

\section{Barriers to receiving surgery}

Of those who received a referral for surgery (97), $35 \mathrm{did}$ not receive surgery, and of those 32 patients reported specific barriers to obtaining surgery to HC providers $(33 \%)$, representing a third delay in accessing surgical care (table 4). The most common specific barriers encountered were the patients not being decision makers to have surgery, ${ }^{7}$ lack of family or social support ${ }^{6}$ and inability to afford hospital fees. ${ }^{5}$ Less commonly the patients expressed fear of surgery, ${ }^{4}$ had no one to accompany them to the hospital ${ }^{4}$ or lacked information about surgery. ${ }^{2}$ Rarely reported was lack of time, fear of lost wages, poor roads to hospital or lack of understanding disease severity. Most patients travelled by ambulance $(46.4 \%)$ or car $(39.2 \%)$ to the hospital. Few were transported by animal $(4.1 \%)$.

\section{DISCUSSION}

It is well established that surgical care throughout Ethiopia is critically limited. However, specific barriers to care and provider and facility needs have not been determined, and the surgical disease burden has not been assessed. This research represents an attempt to further elucidate the barriers to surgical care faced by Ethiopian patients.

In Ethiopia, HCs serve as an important gateway for triaging and referring patients needing essential surgery to higher-level care. The majority of patients requiring surgical care in Ethiopia, particularly in rural settings, can access surgical services first by seeking care at a nearby HC. However, a number of factors related to patients, healthcare providers and material resources at HCs can contribute to the 'three delays' of accessing appropriate surgical care. 


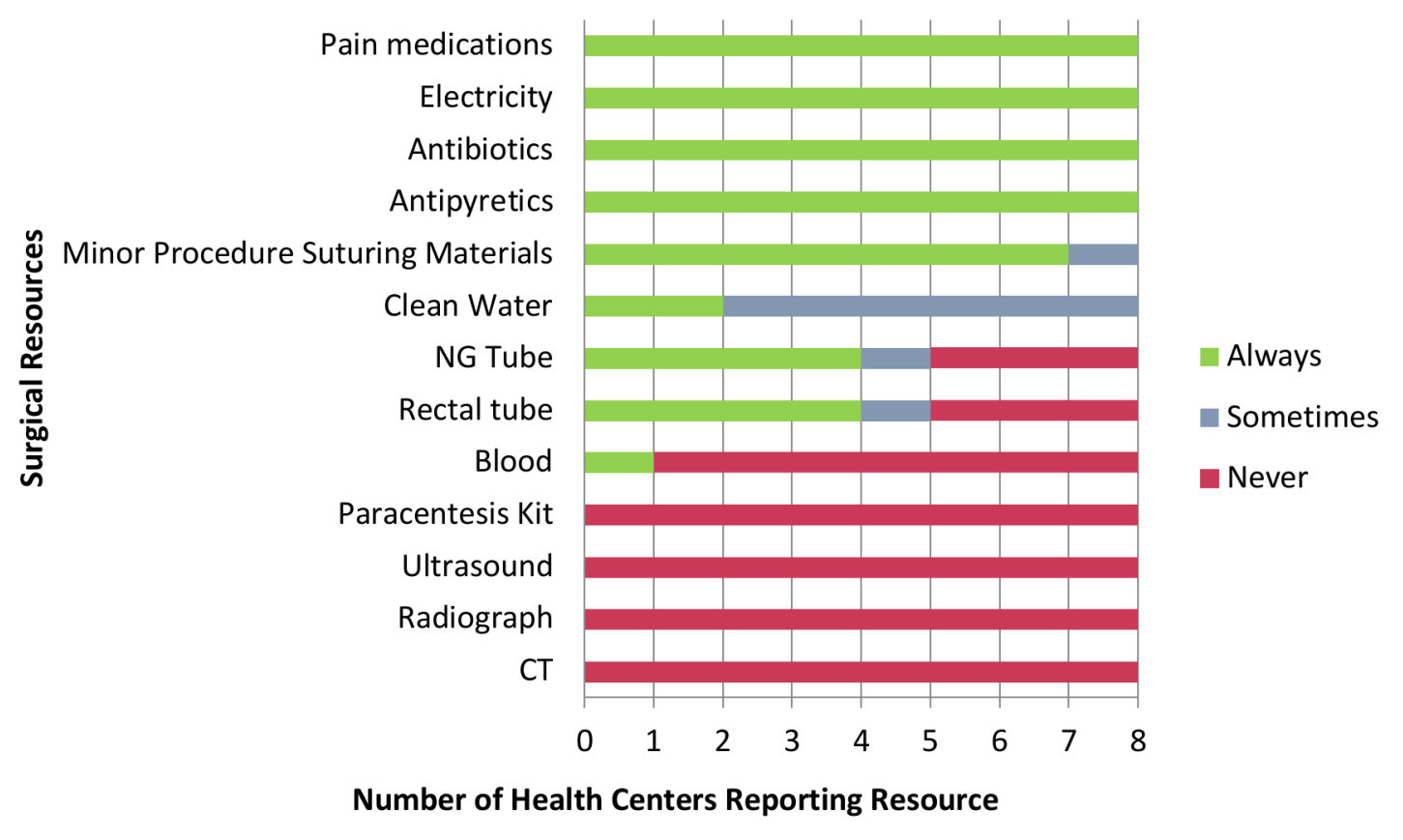

Figure 2 Availability of resources at health centres.

The first delay: patient barriers to care

Barriers experienced by patients can contribute to the first delay; in our study this delay was overcome as all patients presented to HCs, but patient barriers related to decision-making capability and financial resources may still contribute to the 'first delay' in the decision to seek definitive surgical care at the referral hospital.

Many of the patients seen at HCs in our study were perceived to require surgery by the clinical judgement of the HC providers, but ultimately not referred to a surgeon

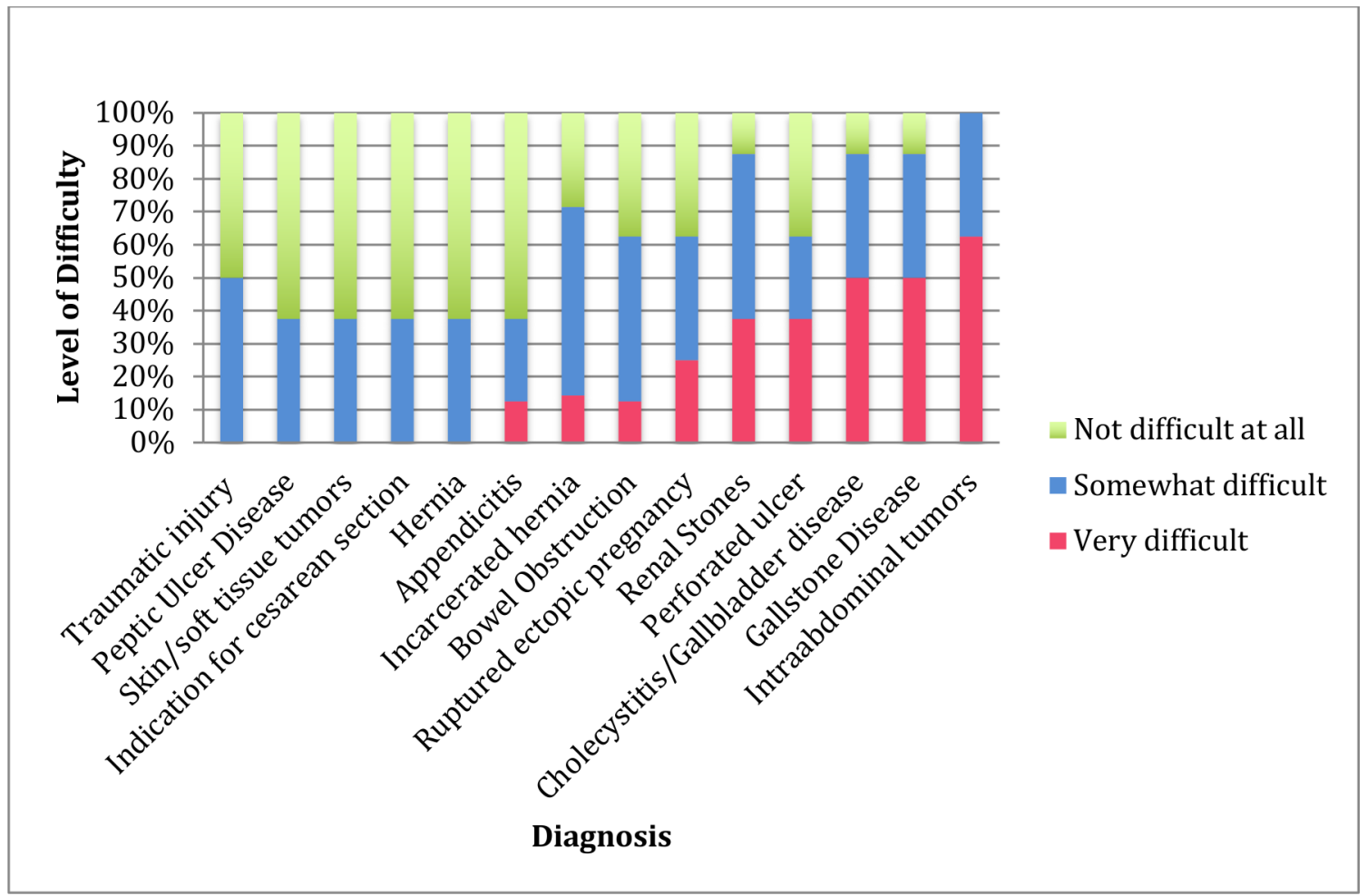

Figure 3 Difficulty in making diagnoses. 
Table 2 Reasons provided by health centre staff for difficulty in making diagnoses $(n=8)$

\begin{tabular}{llll}
\hline & \multicolumn{3}{l}{ Reason for difficulty } \\
\cline { 2 - 4 } Diagnosis & $\begin{array}{l}\text { Lack of } \\
\text { diagnostic } \\
\text { aids }\end{array}$ & $\begin{array}{l}\text { Lack of } \\
\text { training }\end{array}$ & $\begin{array}{l}\text { Atypical } \\
\text { presentation }\end{array}$ \\
\hline Traumatic injury & 4 & - & - \\
\hline Incarcerated hernia & 2 & 1 & 1 \\
\hline Appendicitis & 2 & - & 1 \\
\hline Gallbladder disease & 6 & - & - \\
\hline Bowel obstruction & 4 & 1 & - \\
\hline $\begin{array}{l}\text { Indication for caesarean } \\
\text { section }\end{array}$ & 1 & 2 & - \\
\hline $\begin{array}{l}\text { Perforated ulcer } \\
\text { Ruptured ectopic }\end{array}$ & 5 & - & - \\
pregnancy & 5 & - & - \\
\hline Hernia & 2 & - & 2 \\
\hline Renal stones & 4 & 1 & \\
\hline $\begin{array}{l}\text { Intra-abdominal } \\
\text { tumours }\end{array}$ & - & - & - \\
\hline $\begin{array}{l}\text { Peptic ulcer disease } \\
\text { Skin/soft tissue tumours }\end{array}$ & 4 & - & - \\
\hline
\end{tabular}

for their diagnoses. While at times minor injuries could be treated in the HC, about half of patients referred to the zonal hospital for surgery did not ultimately go to the hospital to pursue surgical care. In order to meet

\begin{tabular}{lc}
\hline $\begin{array}{l}\text { Table } 3 \text { Baseline characteristics of surgical patients at } \\
\text { district health centres }(n=168)\end{array}$ & N (\%) \\
\hline Baseline characteristics & $34.2 \pm 16.8$ \\
\hline Age (mean \pm SD) & $107(63.6)$ \\
Sex & $61(36.3)$ \\
$\quad$ Male & \\
Female & $97(57.7)$ \\
Referral for surgery & $71(42.2)$ \\
Yes & \\
No & $62(87.3)$ \\
Reason for lack of referral $(n=71)$ & $9(12.7)$ \\
Minor injury treated in clinic & \\
Refused referral & $49(50.5)$ \\
Referred patients receiving surgery $(n=97)$ & $35(36.1)$ \\
Yes & $13(13.4)$ \\
No & \\
Unknown & $45(46.4)$ \\
Transportation to hospital & $38(39.2)$ \\
Ambulance & $4(4.1)$ \\
Car & $10(10.3)$ \\
Animal & \\
Not reported &
\end{tabular}

Ethiopian Ministry of Health and WHO targets for essential surgical care, patients should be triaged, appropriately diagnosed and able to reach Dessie Referral Hospital within 2 hours to receive Bellwether or other emergency and essential surgical procedures. ${ }^{23}$ Limitations in diagnostic capabilities, provider training and patient barriers all can contribute to delays in receiving appropriate care, ${ }^{1516}$ which in South Wollo Zone would require transport to the referral hospital for most surgical disease.

The two most common reasons cited for not being referred were that the patient was not the primary decision maker and lacked family support. This underscores the importance of the family in medical decision-making that must be considered when designing interventions to improve healthcare access. What is lacking from these data is an understanding of why a family might withhold support for seeking surgical care. The next most commonly cited reason for refusing a referral was inability to afford the surgery. Whether a lack of familial support for surgery also stems from financial concerns, or if there are other factors weighing in on a family's decision needs to be investigated further. Of note, male patients were far more likely to arrive at the HCs than were female patients (92 male patients and 54 female patients), however men were more likely to be injured as well. These barriers of inadequate social or family support ${ }^{24}$ and financial concerns are echoed in the worldwide literature. ${ }^{25}$ However the barrier of being considered the 'decision maker' for surgery was unique to this context and may be important to explore community and family dynamics to understand further.

One future area for research is further elucidating the reasons behind the lack of family support for patients citing this barrier as a reason for not seeking surgical care. Should this stem from monetary reasons, financial interventions may be additionally required. Future research should also focus on the effects of the SaLTS programme on enabling surgical patients to receive appropriate care, and on the barrier variability across different areas in Ethiopia. Additionally, the sociocultural barriers such as patient autonomy, family and social support should be explored further to better understand healthcare seeking behaviour of rural Ethiopian populations.

\section{The 'third delay': accessing appropriate surgical care}

Importantly, the data collected suggest severe limitations with performing even minor procedures at the HCs. While all centres reported that they always had access to electricity, pain medication, antibiotics and antipyretics, only some had consistent access to clean water, NG tubes, rectal tubes, and none had available imaging equipment. Only one centre reported having access to blood. According to international standards, these facilities aim to provide 'HC' level care, with access to minor surgery, basic medical care, family planning and safe childbirth services. ${ }^{22}$ With inconsistent availability of clean water, rectal tubes, NG tubes and suturing kits, patients who are able to reach the HC still may not receive needed care. 


\section{South Wollo Zone Health Centers: Surgical Patient Diagnoses} $(n=168)$

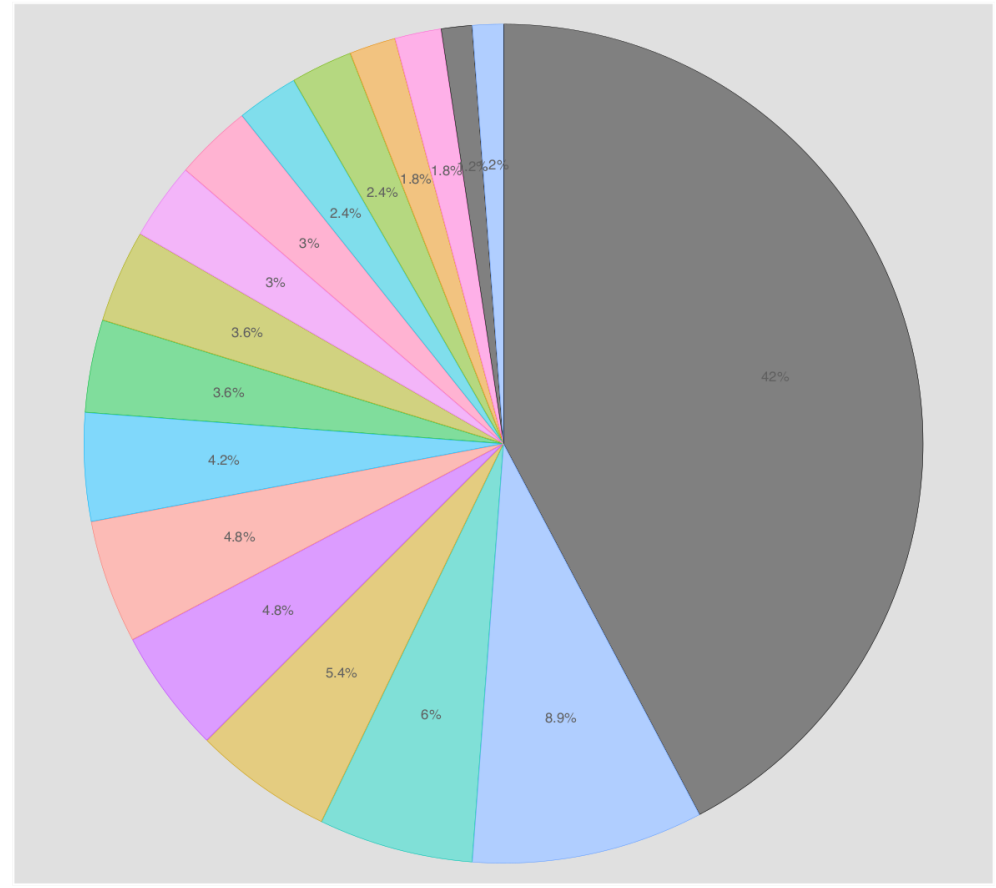

Trauma/Burn

Cesearean Indication

Peptic Ulcer Disease

Bowel Obstruction

Unknown

Appendicitis

$\mathrm{BPH}$

Abscess/Soft Tissue Infection

Circumcision or other urologic

Renal/Bladder stone

Soft Tissue Tumor

Goiter

Hernia

Hemorrhoids

Uterine Prolapse or Myoma

Breast Mass

Gallbladder Disease

Figure 4 South Wollo Zone health centres: surgical patients diagnoses.

For many patients, the only way to receive even minor surgery or emergency procedures may be to have the means and ability to travel to a hospital for care. Some studies have supported the decentralisation of surgical care to rural areas by task-shifting to non-physician surgical providers or surgical 'camps' to provide

\section{Table 4 Barriers to receiving surgery $(n=35)$}

\begin{tabular}{|c|c|c|}
\hline \multicolumn{3}{|l|}{ Patient factors } \\
\hline $\begin{array}{l}\text { Patient is not the decision maker to have } \\
\text { surgery }\end{array}$ & 7 & $20.0 \%$ \\
\hline Lack of family or social support & 6 & $17.1 \%$ \\
\hline No one to accompany patient to surgery & 4 & $11.4 \%$ \\
\hline $\begin{array}{l}\text { Fear of surgery, anaesthesia or bad } \\
\text { outcomes }\end{array}$ & 4 & $11.4 \%$ \\
\hline $\begin{array}{l}\text { Lack of information about disease, } \\
\text { process of surgery or postoperative care }\end{array}$ & 2 & $5.7 \%$ \\
\hline Lack of time & 1 & $2.9 \%$ \\
\hline $\begin{array}{l}\text { Patient does not understand severity of } \\
\text { condition }\end{array}$ & 1 & $2.9 \%$ \\
\hline
\end{tabular}

\section{Financial factors}

\begin{tabular}{lll}
$\begin{array}{l}\text { Patient cannot afford hospital fees, } \\
\text { transportation charge }\end{array}$ & 5 & $14.3 \%$ \\
$\begin{array}{l}\text { Patient cannot afford to lose wages } \\
\text { during surgery/recovery }\end{array}$ & 1 & $2.9 \%$ \\
Structural factors & & \\
Poor roads & 1 & $2.9 \%$ \\
None reported & 3 & $8.6 \%$ \\
\hline
\end{tabular}

intermittent services. ${ }^{26}$ However given the resource limitations at primary hospitals in Ethiopia, ${ }^{27}$ this solution may not be reasonable in this setting. Additionally, the Ethiopian $\mathrm{MOH}$ has continued to build new hospitals and train non-physician surgical providers (IESOs) in the interim, therefore some of these delays may be expected to improve, although these mid-level providers staff hospitals and usually not HCs.

Most or all centres reported difficulty diagnosing perforated ulcer, gallstones, cholecystitis and renal stones, all of which are diagnoses typically confirmed through the very imaging modalities these centres lack. Unsurprisingly, healthcare workers identified a lack of diagnostic aids as the number one barrier to making definitive diagnoses in their centres. None of the eight centres ever had access to radiographs or ultrasound. The general pattern of resource availability demonstrates that HCs are better equipped for managing infectious and communicable diseases than they are for making diagnoses that require imaging equipment. Difficulties in diagnoses contribute to the 'third' delay in care, that is a delay in accessing appropriate care. ${ }^{15} 16$ While this study was unable to follow patients to their encounters at the surgical referral hospital, further study may be useful to determine the impact of such delays on patient outcomes.

Studies have demonstrated that ultrasound can be effectively introduced into such limited-resource settings with success. ${ }^{28}$ This presents a possible mode of intervention to improve diagnosis and referral decisions in South Wollo HCs. Basic ultrasonography can potentially aid in diagnosis and management of soft tissue infections, 
intra-abdominal pathologies, bleeding in trauma and obstetric complications. While definitive management for such conditions would not be expected at the HC level, ultrasound may be a helpful aid in increasing efficiency of diagnosis and triage of patients who need hospital-level care. Of note, however, introducing ultrasound equipment to HCs will require more than the purchase of ultrasound machines. Dedicated intensive ultrasound training would likely need to take place for this intervention to be successful in improving $\mathrm{HC}$ diagnostics. While this is one possible avenue reported by providers at HCs for further training, emphasis should be placed on provision of basic resources, such as clean water, suturing materials, NG and rectal tubes for urgent decompression, and the like, prior to a large-scale investment in resources like ultrasound or radiograph which require additional training and sometimes personnel for use and interpretation.

\section{Framing the study within the context of SaLTS}

Ethiopia is currently implementing the SaLTS programme, a national surgical plan to improve access to and quality of surgery nationwide. This plan which encompasses human resource and infrastructure development, data quality, service quality and monitoring and evaluation will be crucial for closing gaps in access to surgical care. ${ }^{14}$ As part of the SaLTS initiative, an evaluation of surgical hospitals in two regions, one of which encompasses this study's HCs, revealed that access to surgical care may be limited by difficult roads and delays in transport, as well as limitations of all five basic domains of surgical care (service delivery, infrastructure, workforce, information management, financing). ${ }^{27}$

Suboptimal road conditions and hospital resource limitations may be time-consuming and costly to address, however the patient barriers, healthcare provider resource and training constraints may also contribute to delays and offer important context to the ongoing implementation of the SaLTS programme, particularly as it relates to HCs.

\section{LIMITATIONS}

This study had several limitations: data collection time periods varied across sites, and different farming or weather patterns may have affected the number of patients presenting to HCs throughout the year as well as their presenting diagnoses, such as work-related injuries or road traffic accidents. The survey completion rate was low, with $40 \%$ of HCs in the zone completing surveys, therefore the data may not be representative of the entire South Wollo Zone. Also, small numbers of patients reported which barriers were affecting their decision or ability to be referred for surgery and, while descriptive, were not reported in large enough numbers to draw larger conclusions about the population.

Additionally, there are wide variations in culture and economic prosperity between regions in Ethiopia, which may affect healthcare seeking behaviour and perceived barriers to care. This study was conducted in the South
Wollo Zone of Amhara region, Ethiopia, and therefore findings may not necessarily be applicable to other regions in Ethiopia.

\section{CONCLUSION}

This study represents a contribution to the literature as the only survey of $\mathrm{HC}$ resources and diagnostic capabilities in Ethiopia, and of rural populations that may often present with surgical complaints but fail to reach a hospital with surgical capacity due to sociocultural or financial reasons. This study must be taken in context with the ongoing Ethiopian National Surgical Plan, which clearly sets the stage for any evaluation and change in terms of surgical services in Ethiopia, but demonstrates that HCs may lack essential resources and training needed to treat and triage surgical patients. Community and patient barriers must also be mitigated as much as possible to ensure that surgery is acceptable and affordable when needed.

Contributors NS conceived and designed the study and survey tool with input from GK and MW. NS, SC, AS, RC and MW participated in data collection and interpretation of data. NS, SC and MC conducted data analysis. NS and SC drafted the manuscript. SC, AS, MC, GK and MW critically revised the manuscript and approved final version of the paper.

Funding This study was funded by the Massachusetts Medical Society International Health Studies Grant.

Map disclaimer The depiction of boundaries on the map(s) in this article does not imply the expression of any opinion whatsoever on the part of BMJ (or any member of its group) concerning the legal status of any country, territory, jurisdiction or area or of its authorities. The map(s) are provided without any warranty of any kind, either express or implied.

Competing interests None declared.

Patient consent for publication Not required.

Ethics approval Institutional Review Board approval was obtained from Boston University (Boston, MA, USA) and Wollo University (Dessie, Ethiopia) prior to survey distribution.

Provenance and peer review Not commissioned; externally peer reviewed.

Data availability statement All data relevant to the study are included in the article or uploaded as supplementary information.

Open access This is an open access article distributed in accordance with the Creative Commons Attribution Non Commercial (CC BY-NC 4.0) license, which permits others to distribute, remix, adapt, build upon this work non-commercially, and license their derivative works on different terms, provided the original work is properly cited, appropriate credit is given, any changes made indicated, and the use is non-commercial. See: http://creativecommons.org/licenses/by-nc/4.0/.

ORCID iD

Nichole Starr http://orcid.org/0000-0003-0714-5512

\section{REFERENCES}

1 Rose J, Chang DC, Weiser TG, et al. The role of surgery in global health: analysis of United States inpatient procedure frequency by condition using the global burden of disease 2010 framework. PLoS One 2014;9:e89693.

2 Fehlberg T, Rose J, Guest GD, et al. The surgical burden of disease and perioperative mortality in patients admitted to hospitals in Victoria, Australia: a population-level observational study. BMJ Open 2019;9:e028671.

3 Debas HT, Donkor P, Gawande A, et al. Disease control priorities. In: Essential surgery [Internet] Volume 1. 3rd edn. The World Bank, 2015: 442. https://elibrary.worldbank.org/doi/abs/10.1596/978-14648-0346-8 
4 Weiser TG, Haynes AB, Molina G, et al. Size and distribution of the global volume of surgery in 2012. Bull World Health Organ 2016;94:201-9.

5 Meara JG, Leather AJM, Hagander L, et al. Global surgery 2030: evidence and solutions for achieving health, welfare, and economic development. Int J Obstet Anesth 2016;25:75-8.

6 Chao TE, Burdic M, Ganjawalla K, et al. Survey of surgery and anesthesia infrastructure in Ethiopia. World J Surg 2012;36:2545-53.

7 LeBrun DG, Chackungal S, Chao TE, et al. Prioritizing essential surgery and safe anesthesia for the post-2015 development agenda: operative capacities of 78 district hospitals in 7 low- and middleincome countries. Surgery 2014;155:365-73.

8 Weiser TG, Regenbogen SE, Thompson KD, et al. An estimation of the global volume of surgery: a modelling strategy based on available data. Lancet 2008;372:139-44.

9 Reshamwalla S, Gobeze AA, Ghosh S, et al. Snapshot of surgical activity in rural Ethiopia: is enough being done? World J Surg 2012;36:1049-55.

10 Grimes CE, Bowman KG, Dodgion CM, et al. Systematic review of barriers to surgical care in low-income and middle-income countries. World J Surg 2011;35:941-50.

11 Farmer PE, Kim JY. Surgery and global health: a view from beyond the OR. World J Surg 2008;32:533-6.

12 Levine AC, Presser DZ, Rosborough S, et al. Understanding barriers to emergency care in low-income countries: view from the front line. Prehosp Disaster Med 2007;22:467-70.

13 Ethopian Standard. Health center-requirements [Internet]. Available: http://www.forsslund.org/StandardHealthFaclitiy/Health\%20Center. pdf [Accessed 2 May 2019].

14 Burssa D, Teshome A, Iverson K, et al. Safe surgery for all: early lessons from implementing a national government-driven surgical plan in Ethiopia. World J Surg 2017;41:3038-45.

15 Barnes-Josiah D, Myntti C, Augustin A. The 'three delays' as a framework for examining maternal mortality in Haiti. Soc Sci Med 1998;46:981-93.

16 Papali A, McCurdy MT, Calvello EJB. A 'three delays' model for severe sepsis in resource-limited countries. J Crit Care 2015;30:861. e9-14.
17 World Health Organization. Ethiopia: country profiles [Internet]. Available: http://www.who.int/gho/countries/eth/country_profiles/en/ [Accessed 9 Apr 2014].

18 Gebrehiwot T, San Sebastian M, Edin K, et al. Health workers perceptions of facilitators of and barriers to institutional delivery in Tigray, Northern Ethiopia. BMC Pregnancy Childbirth 2014;14:137.

19 Zafar SN, Fatmi Z, lqbal A, et al. Disparities in access to surgical care within a lower income country: an alarming inequity. World $J$ Surg 2013;37:1470-7.

20 Hang HM, Byass P. Difficulties in getting treatment for injuries in rural Vietnam. Public Health 2009;123:58-65.

21 Irfan FB, Irfan BB, Spiegel DA. Barriers to accessing surgical care in Pakistan: healthcare barrier model and quantitative systematic review. J Surg Res 2012;176:84-94.

22 Watkins DA, Jamison DT, Mills A. Chapter 3 Universal health coverage and essential packages of care. In: Jamison DT, Gelband $\mathrm{H}$, Horton S, eds. Disease control priorities: improving health and reducing poverty [Internet]. 3rd edn. Washington, DC: The International Bank for Reconstruction and Development / The World Bank, 2017. http://www.ncbi.nlm.nih.gov/books/NBK525285/

23 O'Neill KM, Greenberg SLM, Cherian M, et al. Bellwether procedures for monitoring and planning essential surgical care in low- and middle-income countries: caesarean delivery, laparotomy, and treatment of open fractures. World J Surg 2016;40:2611-9.

24 Geneau R, Lewallen S, Bronsard A, et al. The social and family dynamics behind the uptake of cataract surgery: findings from Kilimanjaro region, Tanzania. Br J Ophthalmol 2005;89:1399-402.

25 Ologunde R, Maruthappu M, Shanmugarajah K, et al. Surgical care in low and middle-income countries: burden and barriers. Int $J$ Surg 2014;12:858-63.

26 Atiyeh BS, Gunn SWA, Hayek SN. Provision of essential surgery in remote and rural areas of developed as well as low and middle income countries. Int J Surg 2010;8:581-5.

27 Iverson KR, Garringer K, Ahearn O, et al. Mixed-methods assessment of surgical capacity in two regions in Ethiopia. $\mathrm{Br} J$ Surg 2019;106:e81-90.

28 Sippel S, Muruganandan K, Levine A, et al. Review article: use of ultrasound in the developing world. Int J Emerg Med 2011;4:72. 